\title{
The impact of pertussis in infants: insights from a hospital-based enhanced surveillance system, Lazio region, Italy, 2016 to 2019
}

Elisabetta Pandolfi ${ }^{1}$, Francesco Gesualdo ${ }^{1}$, Caterina Rizzo ${ }^{1}$, Luisa Russo ${ }^{1}$, Ilaria Campagna ${ }^{1}$, Emanuela Carloni ${ }^{1}$, Carlo Concato ${ }^{2}$, Giulia Linardos ${ }^{2}$, Alberto Villani ${ }^{3}$, Sara Ciampini ${ }^{1}$, Antonino Reale ${ }^{4}$, Elena Boccuzzi ${ }^{4}$, Fabio Midulla ${ }^{5}$, Alberto E Tozzi ${ }^{1}$

1. Bambino Gesû̀ Children's Hospital, IRCCS, Multifactorial and Complex Disease Research Area, Rome, Italy

2. Bambino Gesù Children's Hospital, IRCCS, Virology Unit, Rome, Italy

3. Bambino Gesù Children's Hospital, IRCCS, Paediatric and Infectious Diseases Unit, Rome, Italy

4. Bambino Gesù Children's Hospital, IRCCS, Emergency Department, Rome, Italy

5. Sapienza University, Department of Paediatrics, Rome, Italy

\section{Correspondence: Francesco Gesualdo (francesco.gesualdo@opbg.net)}

Citation style for this article:

Pandolfi Elisabetta, Gesualdo Francesco, Rizzo Caterina, Russo Luisa, Campagna Ilaria, Carloni Emanuela, Concato Carlo, Linardos Giulia, Villani Alberto, Ciampin Sara, Reale Antonino, Boccuzzi Elena, Midulla Fabio, Tozzi Alberto E. The impact of pertussis in infants: insights from a hospital-based enhanced surveillance system, Lazio region, Italy, 2016 to 2019. Euro Surveill. 2021;26(24):pii=2000562. https://doi.org/10.2807/1560-7917.ES.2021.26.24.2000562

Background: Routine surveillance systems for pertussis often suffer from under-recognition and under-reporting. Aim: Our aim was to describe the epidemiology and the clinical features of pertussis in childrenyounger than 1 year in an Italian region, detected through an enhanced hospital surveillance system. Methods: From 2016 to 2019, we monitored the incidence and the clinical characteristics of hospitalised pertussis casesyounger than 1 year in two paediatric hospitals involved in the PERTINENT project. Results: We detected 141 pertussis cases, corresponding to an estimated incidence of 105.8 per 100.000 in $2016,91.7$ per 100.000 in $2017,64.5$ per 100.000 in 2018 and 40.9 per 100.000 in 2019, based on the hospitals' catchment area, roughly corresponding to the Lazio region. A total of 101 cases $(77.1 \%)$ had a household member with cough or other respiratory symptoms. The most frequent combination of symptoms was paroxysmal cough with apnoea in the absence of fever. Almost $40 \%$ had been prescribed an antibiotic treatment before hospitalisation, and the median time from symptom onset to contact with the hospital was 8 days. Thirty-one $(22.0 \%)$ had complications. Conclusion: An enhanced surveillance system showed a high incidence of pertussis among infants in the Lazio region, where the impact of this disease may still be underestimated. Increasing the coverage of pertussis immunisation among pregnant women and improving the capacity for early detection in primary care may contribute to reducing the impact of pertussis among infants.

\section{Introduction}

Pertussis in infants is frequently associated with severe clinical pictures [1]. Unvaccinated infants pay the highest toll in terms of morbidity and mortality for pertussis [2]. The first dose of the pertussis vaccine is usually administered at 2 or 3 months of age and infants are not protected until completion of the first three doses by 11 months of age $[3,4]$.

A deep understanding of the epidemiology of the disease through surveillance is needed to inform effective control measures.

Pertussis incidence figures are strongly heterogeneous across different countries [5-8] and are often not consistent with seroepidemiological studies, which have hypothesised that the true incidence of the disease might be much higher than reported by surveillance systems $[9,10]$.

The difference in incidence may not reflect a true difference in the disease occurrence, as incidence figures can be influenced by a number of factors, mainly related to under-recognition and under-reporting $[6,11]$. Firstly, although the introduction of the RT-PCR laboratory test for confirmation of the diagnosis has recently improved the sensitivity of pertussis surveillance systems in Europe [5], the suspicion of pertussis and the subsequent decision to prescribe a laboratory confirmation test are often based on non-specific clinical signs, which may lead clinicians to suspect other respiratory conditions. Secondly, the pertussis case definitions from the European Centre for Disease Prevention and Control (ECDC) includes prolonged cough [12], 
General characteristics of infant pertussis cases, Lazio, Italy, January 2016-December $2019(\mathrm{n}=141)$

\begin{tabular}{|c|c|c|c|c|c|c|c|}
\hline & \multicolumn{3}{|c|}{$\begin{array}{c}\text { Positive PCR } \\
(n=141)\end{array}$} & \multicolumn{3}{|c|}{$\begin{array}{c}\text { Negative PCR } \\
(n=405)\end{array}$} & \multirow[t]{2}{*}{$\mathrm{p}$ value } \\
\hline & \multicolumn{2}{|c|}{ Median } & Range & \multicolumn{2}{|c|}{ Median } & \multirow{2}{*}{$\begin{array}{c}\text { Range } \\
0.3-12.0\end{array}$} & \\
\hline Age in months & & .6 & $0.4-11.6$ & & .1 & & 0.730 \\
\hline Birth Weight in kg & & .2 & $0.700-4.925$ & & .1 & $0.690-5.020$ & 0.002 \\
\hline \multirow[t]{2}{*}{ Gestational age in weeks } & \multicolumn{2}{|c|}{39} & $27-41$ & \multicolumn{2}{|c|}{38} & $26-42$ & 0.005 \\
\hline & $\mathrm{n}$ & $\%$ & $95 \% \mathrm{Cl}$ & $\mathrm{n}$ & $\%$ & $95 \% \mathrm{Cl}$ & $p$ value \\
\hline Female & 63 & 44.7 & $36.6-52.9$ & 196 & 48.4 & $43.4-53.4$ & 0.447 \\
\hline Preterm infants & 17 & 12.1 & $7.2-18.6$ & 84 & 20.7 & $16.9-25.0$ & 0.022 \\
\hline Working mother & 82 & 58.6 & $49.9-66.0$ & 236 & 58.3 & $53.3-63.1$ & 0.951 \\
\hline Mother with degree & 47 & 33.8 & $25.9-41.4$ & 145 & 36.3 & $31.5-41.2$ & 0.605 \\
\hline Working father & 131 & 93.6 & $87.7-96.3$ & 380 & 94.1 & $88.1-97.0$ & 0.835 \\
\hline Father with degree & 39 & 28.3 & $20.7-35.5$ & 105 & 26.3 & $22.0-30.9$ & 0.645 \\
\hline More than three household members & 105 & 74.5 & $66.8-81.1$ & 317 & 78.3 & $73.9-82.2$ & 0.353 \\
\hline One or more siblings & 96 & 68.1 & $60.0-75.4$ & 305 & $75 \cdot 3$ & $70.8-79.4$ & 0.094 \\
\hline Mother received pertussis vaccine during pregnancy & o & 0 & NA & 3 & 0.7 & $0.2-2.2$ & 0.814 \\
\hline
\end{tabular}

$\mathrm{Cl}$ : confidence interval; NA: not applicable; n.c. not calculable.

Denominators vary because of missing data for the following variables: working mother, mother with degree, working father, father with degree.

which also guides the diagnosis of suspected pertussis. However, based on our clinical experience and on the results shown below, parents of infants often seek care well before 2 weeks from the start of the cough and therefore, cases can easily be misdiagnosed, with a consequent impact on disease reporting.

In order to better inform surveillance and case management strategies, it may be useful to study the epidemiology of the disease and combine incidence data with the factors associated with access to healthcare and with information on the clinical features and the course of the disease.

In 2015, the ECDC funded the Pertussis in Infants European Network (PERTINENT), a hospital-based active sentinel surveillance system to measure the incidence of whooping cough in infants under the age of than 1 year in Europe. PERTINENT currently includes 37 hospitals from seven surveillance sites in the European Union and European Economic Area, including two large paediatric hospitals located in the Lazio region, Italy.

In Italy, the primary immunisation schedule for pertussis includes three doses in the $3 \mathrm{rd}$, 5th and 11th month of age, with booster doses recommended in the 6th year of life, between the 12th and 18th year of life, and every 10 years after that [13]. Since 2017, the Ministry of Health has recommended pertussis immunisation in pregnancy, which has not achieved a high coverage yet.

In this study, we take advantage of the PERTINENT surveillance system to describe the epidemiology of pertussis in an Italian region, its clinical features and the factors affecting the timing of access to healthcare services among patients with pertussis in the first year of life.

\section{Methods}

\section{Study design and setting}

This is an observational study in a population of infants younger than 1 year, hospitalised for pertussis and identified through an enhanced hospital surveillance programme. The study was conducted between 1 January 2016 and 31 December 2019 and included two large hospitals in the metropolitan area of Rome, Lazio region, Italy.

\section{Study population}

Based on the PERTINENT protocol [14], the study population included all infants younger than 1 year accessing the emergency room with the following case definition: apnoea or a cough associated with at least one additional of the following signs: paroxysms, whoop or post-tussive vomiting ('typical' presentation). Patients with none of these typical symptoms were included in the study population if their physician had suspected pertussis ('atypical' presentation).

Patients with these criteria were screened for pertussis with RT-PCR and, when possible, bacterial culture of the nasopharyngeal aspirate. The patients' families were interviewed after signing an informed consent form. We defined a confirmed pertussis case as a patient meeting the above case definition and either a positive RT-PCR for Bordetella pertussis or a positive culture. 


\section{FIGURE}

Cases of pertussis in infants by year and month, Lazio, Italy, January 2016-December $2019(\mathrm{n}=141)$

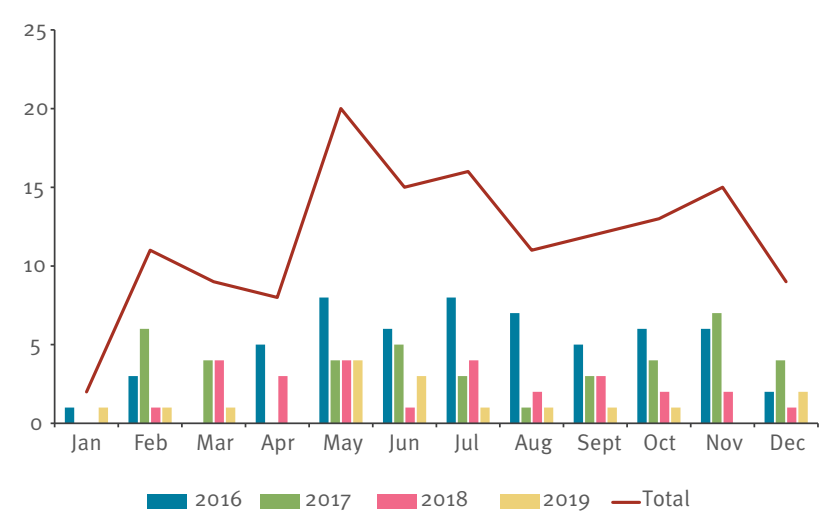

\section{Data collection}

We collected the following data through an interview with cases' parents: sociodemographic data, gestational age, birth weight, level of education and employment of the parents, patient's immunisation status against pertussis through vaccination cards, date of symptom onset, kind of feeding at symptom onset, number of household members and presence of a household member with respiratory symptoms. We also collected the following information during admission: length of stay, complications and admission to the intensive care unit. We also recorded if the patient had both leukocytosis (i.e. white blood cell counts greater than the maximum value for age [15]) and a $>50 \%$ proportion of lymphocytes over the total leukocyte count.

\section{Laboratory confirmation}

Nasopharyngeal aspirates were performed and processed using a standardised protocol [14]. Samples were collected within $24 \mathrm{~h}$ of hospital admission and processed immediately or stored at $-70^{\circ} \mathrm{C}$ until performing the test.

Nasopharyngeal aspirates were immediately spread onto Bordet-Gengou and Regan-Lowe selective agar after being homogenised through vortexing. The plates were incubated in a humidified incubator at $37^{\circ} \mathrm{C}$ for 7 days. Colonies of B. pertussis and B. parapertussis were verified by Gram staining, biochemical tests and MALDI-TOF mass spectrometry.

Nucleic acids were extracted from a $200 \mu \mathrm{L}$ sample of rhinopharyngeal aspirate and purified using the EZ1 Virus Mini Kit v. 2.0 on the EZ1 Advanced XL platform (Qiagen, $\mathrm{GmbH}$, Hilden, Germany). Nucleic acid extracts are eluted into $90 \mu \mathrm{L}$ of buffer and processed immediately. The presence of $B$. pertussis was investigated using a Bordetella Real Time PCR kit targeting IS481 (Bordetella R-gene assay Argene, Biomerieux, Marcy l'Etoile, France). To prevent misdiagnosis of $B$. holmesii as $B$. pertussis, all samples positive for $B$. pertussis are confirmed with a specific real-time PCR assay for $B$. pertussis using the promoter of pertussis toxin $(p t x P)$ gene. We also used the Bordetella Real Time PCR kit (Bordetella Parapertussis R-gene assay Argene, Biomerieux) targeting IS1001 for B. holmesii and B. bronchiseptica.

\section{Statistical analysis}

We estimated pertussis incidence related to the resident population younger than 1 year in the catchment area of the two hospitals [16]. This catchment area was arbitrarily defined as the provinces generating pertussis cases for the period under surveillance.

We compared sociodemographic and clinical characteristics of pertussis cases with patients with a negative RT-PCR. Data are presented as mean and standard deviation (SD), or median and range, or proportion and $95 \%$ confidence intervals (CI), as appropriate. Differences in proportions were evaluated through Fisher's exact test or chi-squared test, as appropriate. The time lag from symptom onset to access to the emergency room and associated factors were studied through Cox proportional hazard models.

Stata 13 software (StataCorp, Texas, United States) was used for statistical analysis.

\section{Ethical statement}

The study was approved by the Bambino Gesù Children's Hospital Ethical Committee (protocol no. 1064_OPBG_2016) and was conducted according to the Declaration of Helsinki, 2013 [17].

\section{Results}

\section{Characteristics of infant pertussis cases and their families}

A total of 153 infants under 1 year of age were hospitalised with laboratory-confirmed pertussis in the period between 1 January 2016 and 31 December 2019. Twelve were excluded for the following reasons: one patient was excluded because of a time lag of more than 90 days between onset of symptoms and nasopharyngeal aspirate and 11 did not meet the PERTINENT protocol's case definition. Of these, one had no respiratory symptoms but was swabbed for pharyngeal hyperaemia, one had only a cough, four had only a fever, four had both a cough and a fever, and one had a cough and cyanosis; in none of the cases had the family paediatrician suspected pertussis. A total of 141 infants with a positive RT-PCR for $B$. pertussis were finally included in the analysis. Ninety-three of them (65.9\%) also had a positive pertussis culture. No other Bordetella species were detected.

Table 1 presents sociodemographic characteristics of all patients screened for pertussis, by RT-PCR results. There was a slightly higher proportion of male cases (55.3\%). The sociodemographic level was generally high, $12 \%$ were preterm infants and nearly $70 \%$ had siblings. 
TABLE 2

Symptoms observed in infant pertussis cases, Lazio, Italy, January 2016-December $2019(\mathrm{n}=141)$

\begin{tabular}{|c|c|c|c|c|c|c|c|}
\hline & \multicolumn{3}{|c|}{$\begin{array}{c}\text { Positive PCR } \\
(n=141)\end{array}$} & \multicolumn{3}{|c|}{$\begin{array}{c}\text { Negative PCR } \\
(n=405)\end{array}$} & \multirow[t]{2}{*}{$\mathrm{p}$} \\
\hline & $\mathrm{n}$ & $\%$ & $95 \% \mathrm{Cl}$ & $\mathrm{n}$ & $\%$ & $95 \% \mathrm{Cl}$ & \\
\hline Cough & 136 & 96.5 & $92.3-98.7$ & 332 & 82.0 & $77.9-85.6$ & $<0.001$ \\
\hline Paroxysmal cough & 120 & 85.1 & $78.5-90.2$ & 203 & 50.1 & $45.1-55.1$ & $<0.001$ \\
\hline Apnoea & 107 & 75.9 & $68.3-82.4$ & 194 & 47.9 & $42.9-52.9$ & $<0.001$ \\
\hline Cyanosis & 80 & 56.7 & $48.5-64.7$ & 105 & 26.1 & $21.8-30.6$ & $<0.001$ \\
\hline Whooping & 77 & 54.6 & $46.3-62.7$ & 58 & 14.9 & $11.5-18.8$ & $<0.001$ \\
\hline Post-tussive vomiting & 64 & 45.4 & $37.3-56.7$ & 151 & 37.3 & $32.6-42.2$ & 0.090 \\
\hline WBC $>\max$ for age and $L Y M>50 \%$ & 43 & 31.2 & $23.8-39.2$ & 28 & 7.2 & $4.8-10.2$ & $<0.001$ \\
\hline Fever & 32 & 22.7 & $16.3-30.1$ & 178 & 44.0 & $39.1-48.9$ & $<0.001$ \\
\hline Conjunctival haemorrhage & 15 & 10.6 & $6.3-16.7$ & 15 & 3.7 & $2.1-6.1$ & 0.002 \\
\hline Petechiae & 14 & 10.0 & $5.8-15.8$ & 16 & 4.0 & $2.3-6.5$ & 0.008 \\
\hline Mother with symptoms & 32 & 24.4 & $17.3-32.7$ & 75 & 19.8 & $15.9-24.2$ & 0.267 \\
\hline
\end{tabular}

Cl: confidence interval; WBC: white blood cells. LYM: lymphocytes.

Denominators vary because of missing data for the following variables: cyanosis, whooping, WBC > max for age and LYM > 50\%, conjunctival haemorrhage, petechiae, mother with symptoms.

Fifty-five of 141 cases (39.0\%) were aged 2 months or younger and were not, therefore, eligible for immunisation. Among the 86 infants eligible for immunisation, 42 had not received any dose of the vaccine, 36 had received only one dose, eight had received two doses and none had received three doses. None of the mothers of cases had received pertussis immunisation during pregnancy. Among the 131 cases for whom this information was available, 101 (77.1\%) had one or more relatives with cough or other respiratory symptoms. The most frequent categories were siblings $(n=52$; $39.7 \%)$ and mothers $(n=32 ; 24.4 \%)$. Only two of 52 infant cases with symptomatic siblings had siblings who had not received any dose of pertussis immunisation, while symptomatic siblings of 44 of 52 cases had received at least three doses.

Compared with non-cases (i.e. patients with a nonpertussis respiratory infection), pertussis cases were born at a significantly higher gestational age and with a higher birth weight and were less frequently preterm.

\section{Epidemiology}

The distribution of cases by month and year is reported in the Figure. We observed a peak in incidence rates in 2016 with a subsequent decreasing trend.

The catchment area of the two hospitals, defined as the provinces generating cases in the study period, included all provinces of the Lazio region, except for the province of Rieti, which accounted for less than $2.5 \%$ of the population younger than 1 year in the whole region. Therefore, in the rest of the paper, to perform comparisons with official surveillance data (which are reported by region and not by province) we will consider the catchment area of the two hospitals as an area roughly corresponding to the whole Lazio region. Based on the catchment area, the estimated incidence was 105.8 per 100.000 in 2016 , 91.7 per 100.000 in $2017,64.5$ per 100.000 in 2018 and 40.9 per 100.000 in 2019. The majority of cases were observed between May and September, with lower figures in winter.

\section{Clinical presentation}

Cough, paroxysmal cough and apnoea were the most frequent symptoms (Table 2). The most frequent pattern of symptoms was a combination of paroxysmal cough and apnoea in the absence of fever. These three criteria were simultaneously recorded in 76 (53.9\%) cases. Forty-three (31\%) cases had leukocytosis and lymphocytosis. A total of 56 infants (39.7\%) had received an antibiotic prescription before admission to the hospital. Of these, 30 were prescribed a macrolide, while the remaining 26 received either amoxicillin or an oral cephalosporin.

Cough, paroxysmal cough, apnoea, cyanosis, whooping, leukocytosis and lymphocytosis, conjunctival haemorrhage and petechiae were significantly more frequent in pertussis cases compared with non-cases. Fever was more frequently reported among non-cases.

\section{Clinical course during admission}

The median time to access the emergency room after symptom onset was 8 days (range: $1-53$; interquartile range: 3-14). Apnoea and having had an antibiotic prescription for the current respiratory infection were significantly associated with a shorter time between symptom onset and hospitalisation in the emergency room (apnoea: hazard ratio $(\mathrm{HR})=0.61,95 \% \mathrm{Cl}$ : $0.40-$ 0.93; antibiotics: $H R=0.55,95 \% \mathrm{Cl}: 0.38-0.80)$. The median length of stay was 7 days (range: 1-111).

Thirty-one children (22.0\%) had complications during admission, including the following: hypoxaemia $(n=13)$, difficult feeding $(n=11)$, dehydration $(n=3)$, bacterial 
superinfection $(n=2)$, seizures $(n=2)$, cerebral haemorrhage $(n=1)$, rectal prolapse $(n=1)$, rib fractures $(n=1)$ and acute renal failure $(n=1)$. Nine cases were admitted to the intensive care unit, none died.

The clinical presentation of the disease was similar in term and preterm infants, although complications were more frequent in the latter group. Specifically, dehydration ( $2 / 17$ vs $1 / 124, p<0.01)$ and convulsions (2/17 vs $0 / 124, p<0.01$ ) were observed more frequently in preterm infants with pertussis.

\section{Discussion}

Studying the epidemiology and the impact of pertussis in infants younger than 1 year is crucial to plan appropriate prevention strategies at the local level. Through enhanced surveillance, we found an incidence of pertussis in an Italian region that was higher than that estimated from a sentinel surveillance system in Italy in 2008 [18]. Although pertussis immunisation coverage in Italy exceeds $95 \%$ in the first 2 years of life [19] and the Ministry of Health has recommended immunisation in pregnancy since 2017 [13], pertussis cases still occur among infants too young to be vaccinated.

Although pertussis vaccination coverage across Europe is relatively homogeneous, incidence figures in Europe vary, mainly owing to differences in surveillance systems, case definitions and methods for diagnosis, which consequently affect disease reporting. In one study examining surveillance data from several European countries in 2017, the largest number of pertussis cases were reported in Germany, the Netherlands, Poland, Spain and the United Kingdom (UK), but there were substantial variations across countries [5]. In the same report, infants were the most frequently affected age group, with the exception of the Netherlands, Slovenia and Norway. According to ECDC data, European countries had in 2017 an average incidence of 53.9 cases per 100,000 in infants below 1 year of age, with $50 \%$ of cases among infants aged 3 months or younger [8]. The incidence estimated in infants in our study was 91.7 per 100,000 in 2017, i.e. nearly twice as high, in the same year. Routine surveillance data for the Lazio region are available for the $0-3$ year-old group only and indicate an incidence of 31.7 and 29.0 per 100.000 in 2016 and 2017, respectively [20].

If the results of our study are applied to the rest of the country, it is likely that Italy is impacted by significant under-reporting for pertussis. The temporal trend of pertussis showed an incidence peak in 2016 with a decreasing trend in the following years. The peak (and its subsequent decline) was not associated with outbreaks or with major modification of immunisation policies. Therefore, it can be interpreted as a normal variation in the usual frame of the pertussis epidemic cycle [18].
Twelve per cent of the enrolled population were preterm babies. Taking into account that $7 \%$ of Italian children are estimated to be born preterm [21], there is an over-representation of this subgroup among infants hospitalised for pertussis. Although this result should be interpreted with caution given the wide $95 \% \mathrm{Cl}$ of the proportion, it is in line with previous observations conducted on larger populations in the UK (10.6\% prevalence of preterm infants among infants hospitalised for pertussis) [22] and in Norway (10\%) [23]. The clinical picture of preterm infants was slightly worse compared with term infants, with a higher incidence of dehydration and seizures, while other symptoms, other complications and the length of stay were similar between the two groups.

Under-recognition of pertussis is also an issue. Although a significant proportion of cases in our study presented with symptoms commonly associated with pertussis, others had non-specific signs that resembled other respiratory infections. Moreover, almost $40 \%$ of enrolled cases accessed the emergency room after a symptom duration shorter than 1 week. Interestingly, more than one third of pertussis cases had received an antibiotic prescription before accessing the emergency room, which was a macrolide in only ca $50 \%$ of cases. This may indicate that in almost half of the infants on antibiotic treatment, the physician had not suspected a diagnosis of pertussis. These data are in line with the small likelihood of suspecting pertussis detected in a sample of Italian paediatricians and physicians back in 2013 [24]. Moreover, the severity of the clinical picture affected the time from symptom onset to access to the emergency room, as this time was shorter in infants with apnoea or an antibiotic prescription.

Early recognition of pertussis may benefit from the availability of tools for pertussis diagnosis in primary care. Point-of-care diagnostic tests are not commonly available yet, but ongoing research in this field is promising [25]. The potential clinical impact of tools for point-of-care diagnosis in primary care is well recognised [26], as they may improve the estimation of the impact of pertussis and the management of the disease, avoiding unnecessary antibiotic prescription in case of viral infections and allowing early, appropriate antibiotic treatment to prevent secondary cases.

The impact of pertussis observed in this study may be at least partially preventable. As shown in other studies, the efficacy estimate for only one dose of pertussis vaccine is limited. Moreover, timeliness of immunisation start is crucial, as, according to our results, a large proportion of cases had not yet started their vaccination course although they were eligible. Immunisation during pregnancy has been proven to be safe, effective and cost-effective in preventing pertussis hospitalisations in infants $[27,28]$. Although the Italian vaccine programme includes a recommendation for immunising pregnant women against pertussis in the third trimester, ideally in the 28th gestational week [13], data on 
immunisation coverage are not available yet. In 2016 and 2017, a small-scale seroepidemiological study showed non-protective titres of antibodies in all tested pregnant women in the south of Italy [29]. Two surveys conducted between 2015 and 2018 showed that fewer than $2 \%$ of interviewed women had received tetanus, diphtheria and acellular pertussis (Tdap) vaccination during pregnancy, and only one third showed willingness to be vaccinated during pregnancy $[30,31]$. Based on these studies, we assume that Tdap vaccination coverage during pregnancy is still poor in Italy. Taking into account the costs of hospitalisation and the fact that severe pertussis cases mostly occur in children too young to be eligible for vaccination, immunisation in pregnancy remains a mainstay of pertussis prevention and should be urgently implemented and promoted, given its efficacy, safety and cost-effectiveness and that it had a higher impact than a cocooning strategy or immunisation before pregnancy [27,32]. Nevertheless, a pertussis vaccination programme in pregnancy may have a limited impact on preventing pertussis among preterm infant (over-represented among infants hospitalised with pertussis), because the opportunity for maternal vaccination is smaller in this particular group, as previously demonstrated in a study conducted in the UK. For this reason, from 2016, the recommendation for maternal vaccination in the UK has been anticipated to be between 20 and 32 weeks of gestational age [22]. In Italy, the timing of the pertussis vaccination in pregnancy may be reviewed accordingly.

This study has a number of limitations. Based on the home addresses of the enrolled patients, we assumed that the catchment area of the participating hospitals roughly corresponded to the whole Lazio region. However, we cannot exclude that our study missed cases who might have accessed other hospitals. In both cases, the real incidence in the region could be higher than what we recorded. Unfortunately, recent regional data by age group are not available, therefore reliable comparisons of our figures with those reported by the routine surveillance system is not possible. However, we have been able to compare our data with national data reported to the ECDC and with historical data.

Pertussis PCR results may be affected by antibiotic treatment lasting at least 5 days [33]. Although more than one third of pertussis cases had received an antibiotic before accessing the hospital - and were still PCR-positive - we may have missed additional cases receiving antibiotics and consequently underestimated the real incidence.

\section{Conclusion}

Our study shows that in the participating hospitals' catchment area, which roughly corresponds to the whole Lazio region, the incidence of pertussis in the first year of life has been high in recent years. Based on our assumptions, figures in the Lazio region in 2017 were almost twice as high as the mean European incidence of pertussis in young infants. It is likely that epidemic cycles will continue to occur in the absence of an increased coverage for the pertussis vaccine among pregnant women. In Italy, maternal immunisation remains a mainstay in pertussis prevention, particularly in preventing severe cases, which often occur in very young infants, and associated costs. To improve routine surveillance of pertussis, better pertussis diagnostic tools, including point-of-care tests in primary care, should be available to avoid misclassification of cases and to timely prescribe appropriate antibiotic therapy to prevent secondary cases.

\section{Acknowledgements}

We are grateful to all patients, their legal tutors, nurses, clinicians, microbiologists and epidemiologists from the study sites, who actively participated in the study. Thanks to the biostatistician Antonino Bella, from the National Institute of Health, for his support in data management; thanks to Dr Veronica Bartolucci for her precious support in patients' follow-up and thanks to Dr Valentina Ferro for her support in patients' enrolment.

Funding: This work was partially supported by PERTINENT (Pertussis in Infants European Network), a network funded by the European Centre for Disease Prevention and Control (Framework contract no. ECDC/2015/017).

\section{Conflict of interest}

None declared.

Authors' contributions

EP contributed to the conceptualisation of the study and wrote the manuscript, FG wrote the manuscript and supervised the final revision, CR contributed to the conceptualisation of the study and revised the manuscript, LR and IC contributed to patients' enrolment and patients' follow up, EC contributed to data curation and performed the statistical analysis, CC and GL performed laboratory analysis, provided microbiological data and contributed to writing the methods section, AV revised the manuscript, SC contributed to patients' follow-up and revised the manuscript and, AR and EB contributed to patients' enrolment, FM revised the manuscript, AET contributed to the conceptualisation of the study and wrote the manuscript.

\section{References}

1. Cherry JD, Wendorf K, Bregman B, Lehman D, Nieves D, Bradley JS, et al. An observational study of severe pertussis in 100 infants $\leq 120$ days of age. Pediatr Infect Dis J. 2018;37(3):2025. https://doi.org/10.1097/INF.0000000000001710 PMID: 28737623

2. World Health Organization. Pertussis vaccines: WHO position paper - September 2015. Wkly Epidemiol Rec. 2015;90(35):43358. PMID: 26320265

3. Halperin BA, Halperin SA. The reemergence of pertussis and infant deaths: is it time to immunize pregnant women? Future Microbiol. 2011;6(4):367-9. https://doi.org/10.2217/fmb.11.15 PMID: 21526936

4. Saadatian-Elahi M, Plotkin S, Mills KHG, Halperin SA, Mclntyre PB, Picot V, et al. Pertussis: Biology, epidemiology and prevention. Vaccine. 2016;34(48):5819-26.

5. Heininger U, André P, Chlibek R, Kristufkova Z, Kutsar K, Mangarov A, et al. Comparative epidemiologic characteristics of pertussis in 10 central and eastern European countries, 
2000-2013. PLoS One. 2016;11(6):e0155949. https://doi. org/10.1371/journal.pone.0155949 PMID: 27257822

6. Tan T, Dalby T, Forsyth K, Halperin SA, Heininger U, Hozbor D, et al. Pertussis across the globe: recent epidemiologic trends from 2000 to 2013. Pediatr Infect Dis J. 2015;34(9):e222-32. https://doi.org/10.1097/INF.0000000000000795 PMID: 26376316

7. Jackson DW, Rohani P. Perplexities of pertussis: recent global epidemiological trends and their potential causes. Epidemiol Infect. 2014;142(4):672-84. https://doi.org/10.1017/ So950268812003093

8. European Centre for Disease Prevention and Control (ECDC). Pertussis. Annual Epidemiological Report for 2017. Stockholm: ECDC; 2019. Available from: https://www.ecdc.europa.eu/ sites/default/files/documents/AER_for_2017-pertussis.pdf

9. Barkoff A-M, Gröndahl-Yli-Hannuksela K, He Q. Seroprevalence studies of pertussis: what have we learned from different immunized populations. Pathog Dis. 2015;73(7):ftv050. https:// doi.org/10.1093/femspd/ftv050 PMID: 26208655

10. Chlibek R, Smetana J, Sosovickova R, Fabianova K, Zavadilova J, Dite P, et al. Seroepidemiology of whooping cough in the Czech Republic: estimates of incidence of infection in adults. Public Health. 2017;150:77-83. https://doi.org/10.1016/j. puhe.2017.05.012 PMID: 28646697

11. Wood N, Mclntyre P. Pertussis: review of epidemiology, diagnosis, management and prevention. Paediatr Respir Rev. 2008;9(3):201-11, quiz 211-2. https://doi.org/10.1016/j. prrv.2008.05.010 PMID: 18694712

12. European Commission. Commission implementing decision (EU) $\mathbf{2 0 1 8 / 9 4 5 ~ o f ~} 22$ June 2018 on the communicable diseases and related special health issues to be covered by epidemiological surveillance as well as relevant case definitions. Official Journal of the European Union. Luxembourg: Publications Office of the European Union. 6 jul 2018:L 170/1. Available from: https://eur-lex.europa.eu/legal-content/EN/TXT/PDF/?uri $=$ CELEX\%3A32018D0945\&from=EN\%29

13. della Salute M. Piano Nazionale per la Prevenzione Vaccinale. [National Vaccine Prevention Plan]. PNPV 2017-2019. Rome: Ministero della Salute; 2017. Italian. Available from: http:// www.salute.gov.it/imgs/C_17_pubblicazioni_2571_allegato.pdf

14. PERTINENT Network. Pertinent Pertussis enhanced surveillance: draft generic protocol v 1.5 Paris: EpiConcept; Jun 2020. Available from: https://sites.google.com/a/epiconcept. $\mathrm{fr} /$ pertinent/documentation-and-references/generic-protocol

15. Kliegman RM, Nelson WE. Nelson textbook of pediatrics. 19th ed. Philadelphia: Elsevier; 2011.

16. Istituto Nazionale di Statistica (Istat). Demografia in cifre. [Demographics in figures]. Rome: Istat. [Accessed: 6 Apr 2020]. Italian. Available from: http://demo.istat.it

17. World Medical Association. World Medical Association Declaration of Helsinki: ethical principles for medical research involving human subjects. JAMA. 2013;310(20):2191-4. https:// doi.org/10.1001/jama.2013.281053 PMID: 24141714

18. Gonfiantini MV, Carloni E, Gesualdo F, Pandolfi E, Agricola E, Rizzuto $\mathrm{E}$, et al. Epidemiology of pertussis in Italy: disease trends over the last century. Euro Surveill. 2014;19(40):20921. https://doi.org/10.2807/1560-7917.ES2014.19.40.20921 PMID: 25323077

19. Vaccinazioni dell'età pediatrica anno 2018 (coorte 2016). Ministero della salute; [cited 2020 Apr 6]. Available from: http://www.salute.gov.it/imgs/C_17_tavole_20_allegati iitemAllegati_o_fileAllegati_itemFile_7_file.pdf

20. Malattie prevenibili da vaccino - Regione Lazio 2005-2017. Servizio Regionale per l'Epidemiologia, Sorveglianza e controllo delle Malattie Infettive; [cited 2020 Apr 6]. Available from: https://www.inmi.it/wp-content/uploads/2018/12/ Malattie-prevenibili-da-vaccino.-Lazio-2005-2017.pdf

21. Certificato di assistenza al parto (CeDAP) Analisi dell'evento nascita - Anno 2016 Direzione Generale della digitalizzazione, del sistema informativo sanitario e della Statistica Ufficio di Statistica; [cited 2020 Sep o9]. Available from: http://www. salute.gov.it/imgs/C_17_pubblicazioni_2881_allegato.pdf

22. Byrne L, Campbell H, Andrews N, Ribeiro S, Amirthalingam $\mathrm{G}$. Hospitalisation of preterm infants with pertussis in the context of a maternal vaccination programme in England. Arch Dis Child. 2018;103(3):224-9. https://doi.org/10.1136/ archdischild-2016-311802 PMID: 28814424

23. Riise $\emptyset R$, Laake I, Vestrheim D, Flem E, Moster D, Riise Bergsaker MA, et al. Risk of Pertussis in Relation to Degree of Prematurity in Children Less Than 2 Years of Age. Pediatr Infect Dis J. 2017;36(5):e151-6. https://doi.org/10.1097/ INF.0000000000001545 PMID: 28403056

24. Gonfiantini MV, Villani A, Gesualdo F, Pandolfi E, Agricola E, Bozzola $E$, et al. Attitude of Italian physicians toward pertussis diagnosis. Hum Vaccin Immunother. 2013;9(7):1485-8. https:// doi.org/10.4161/hv.24734 PMID: 23732898

25. Dou M, Macias N, Shen F, Bard JD, Domínguez DC, Li X. Rapid and accurate diagnosis of the respiratory disease pertussis on a point-of-care biochip. EClinicalMedicine.2019;8:72-7. https:// doi.org/10.1016/j.eclinm.2019.02.008 PMID: 31008450

26. Wirsing von Koenig $\mathrm{CH}$. Pertussis: point-of-care testing in the making. EClinicalMedicine.2019;8:4-5. https://doi. org/10.1016/j.eclinm.2019.02.010 PMID: 31193612

27. Baxter R, Bartlett J, Fireman B, Lewis E, Klein NP. Effectiveness of vaccination during pregnancy to prevent infant pertussis. Pediatrics. 2017;139(5):e20164091. https://doi.org/10.1542/ peds.2016-4091 PMID: 28557752

28. Sandmann F, Jit M, Andrews N, et al. Infant hospitalisations and fatalities averted by the maternal pertussis vaccination programme in England, 2012-2017: Post-implementation economic evaluation. Clin Infect Dis. 2020 Feb;ciaa165.

29. Marchi S, Viviani S, Montomoli E, Trombetta CM. Low prevalence of antibodies against pertussis in pregnant women in Italy. Lancet Infect Dis. 2019;19(7):690. https://doi. org/10.1016/S1473-3099(19)30269-5 PMID: 31250813

30. Agricola E, Gesualdo F, Alimenti L, Pandolfi E, Carloni E, D’Ambrosio A, et al. Knowledge attitude and practice toward pertussis vaccination during pregnancy among pregnant and postpartum Italian women. Hum Vaccin Immunother. 2016;12(8):1982-8. https://doi.org/10.1080/21645515.2016.118 8242 PMID: 27712242

31. D'Alessandro A, Napolitano F, D’Ambrosio A, Angelillo IF. Vaccination knowledge and acceptability among pregnant women in Italy. Hum Vaccin Immunother. 2018;14(7):15739. https://doi.org/10.1080/21645515.2018.1483809 PMID: 29863958

32. Hoshi SL, Seposo X, Okubo I, Kondo M. Cost-effectiveness analysis of pertussis vaccination during pregnancy in Japan. Vaccine. 2018 16;36(34):5133-40.

33. American Academy of Pediatrics. Pertussis (Whooping Cough). In: Kimberlin DW, Brady MT, Jackson MA, Long SS, eds. Red Book: 2018 Report of the Committee on Infectious Diseases. American Academy of Pediatrics; 2018; 620-634.

\section{License, supplementary material and copyright}

This is an open-access article distributed under the terms of the Creative Commons Attribution (CC BY 4.0) Licence. You may share and adapt the material, but must give appropriate credit to the source, provide a link to the licence and indicate if changes were made.

Any supplementary material referenced in the article can be found in the online version.

This article is copyright of the authors or their affiliated institutions, 2021. 
